This is the accepted manuscript of the article, which has been published in

European Journal of Cultural Studies. 2019.

https://doi.org/10.1177/1367549419886021

\title{
Affective labour of creating atmospheres
}

Marjo Kolehmainen and Katariina Mäkinen

\section{Abstract}

In this article, we suggest that the production of atmospheres is a significant form of the capitalisation of affect - the ongoing cultural shift that encompasses diverse ways in which affect itself has become a selling point. We further the discussion of this capitalisation of affect by offering insights into forms of capitalisation that mobilise atmospheres rather than single, individual or clearly identified affects. Through two ethnographic case studies, we examine how affective labour is invested in the co-production of atmospheres during collective events in commercial settings. Audiences or participants are actively involved in the production of atmospheres, both intentionally and unintentionally. This kind of affective labour is collective, intercorporeal and trans-subjective and takes various forms from embodied activities to virtual processes. Furthermore, the co-production of atmospheres is itself a component of atmospheres. The article thus opens up new paths into the study of both affective labour and atmospheres, by providing alternative imaginaries to prevailing notions of affective labour.

Keywords

Affect, affective labour, atmosphere, co-production, ethnography

Tampere University, Finland

Corresponding author(s):

Marjo Kolehmainen, Faculty of Social Sciences, Tampere University, Linna 5084, 33014 Tampere, Finland. Email: marjo.kolehmainen@tuni.fi

Introduction

In this article, we suggest that the production of atmospheres is a significant form of the capitalisation of affect - the ongoing cultural shift that encompasses diverse ways in which affect itself has become a selling point. We further the discussion of this capitalisation of affect by offering insights into forms of capitalisation that mobilise atmospheres rather than single, individual or clearly identified affects. We not only argue that affective labour is employed in the creation of atmospheres, but also emphasise that this kind of labour is collective, intercorporeal and trans-subjective, and takes various forms from embodied activities to virtual processes. Our article opens up new paths into the study of both affective labour and atmospheres, by providing alternative imaginaries to prevailing notions of affective labour. Through two ethnographic case studies, we examine how affective labour is invested in the production of atmospheres during collective events in commercial settings. We further argue that affective labour is a form of economic co-production that shapes and engages subjects through multiple connections and settings.

We consider affect theories a particularly useful point of departure for working with atmospheres. Affect cannot be reduced to subjective feelings; instead, it offers a means to explore relations between bodies, as it foregrounds bodies' capacities to affect and be affected (e.g. Blackman, 2010; Seyfert, 2012). In 
particular, we employ the concept of atmosphere, which challenges the binaries of body/mind, self/other and - as we will argue - labourer/client. Atmospheres always reach beyond individuals. As Seyfert (2012) points out, affects are conventionally seen as located within an individual subject or body, or alternatively as atmospheric forces that operate externally to the body. On the one hand, however, if we regard affects as individual, we fail to account for their collective and trans-subjective nature. On the other, if we regard people as simply caught in atmospheres, we risk seeing individuals as passive objects with little agency. Nor do conceptualisations of this kind explain why different people experience the same atmospheres in different ways: not every individual body is necessarily affected in the same way (Seyfert, 2012; see also Edensor, 2015). Thinking of affect as an effect of entanglements and encounters between human and nonhuman bodies (Seyfert, 2012) enables us to see atmospheres as neither residing outside human bodies nor as simply human-made. Furthermore, atmospheres are not only about the social domain; in addition to human and non-human bodies they also involve matters ranging from space and place to the different configurations of objects and technologies (see Edensor, 2015).

The concept of affective labour is currently used in multiple ways. In order to draw both theoretical and empirical distinctions within the concept (Oksala, 2016), we work with the concept of affective labour through empirical case studies. By using this approach to the concept of affective labour, we are able to rethink labour within the processes of the capitalisation of affect. Atmospheres have not been previously explored in relation to affective labour, even though the ways atmospheres are manufactured are regularly discussed in emerging studies of atmospheres. Atmospheres need to be actively created, and it takes work to sustain them, even though they may make us feel as if we are entering pre-given zones (Wetherell, 2012). Writing about English football matches, Tim Edensor (2015) points out how atmospheres are coproduced by players, match organisers and fans alike. However, we seek to elaborate the processes of coproduction in more detail drawing together affective labour and recent work on atmosphere, in order to explore the co-production processes from a novel perspective.

We draw on understandings of atmospheres as dynamic, changing configurations that can be intentionally modified to the extent that they can be harnessed for social, political or economic purposes (Bille et al., 2015; Sumartojo and Pink, 2018: 3). Organising human and non-human bodies, objects and spaces is a central means by which atmospheres are engineered (see Bille et al., 2015). Often this process of engineering atmospheres is framed as manipulation. However, the term 'manipulation' has negative connotations that overlook the everyday and mundane modifications of affect. It also fails to account for actions that contribute to the making and unmaking of atmospheres beyond intentional planning. We therefore rather speak of collective affective labour, a concept that enables alternative imaginaries that do not attach agency to unidirectional and straightforward processes alone.

We hence seek to reimagine affective labour with the help of recent developments in affect theory. This line of enquiry poses challenges to the conceptualisation of affective labour. If affect is not limited to human emotions, subjective feelings or bodily responses, the 'affective' in affective labour cannot be reduced to immaterial investments by workers, or to feelings that emerge in clients. This is because both conceptualisations miss many of the essential features of affect: affect highlights relations between bodies, as it foregrounds bodies' in/capacities to affect and become affected, which emerge in and through encounters between bodies and things. Hence, we build on the observation that affective labour cannot be reduced to the 'property' of labourers alone (Clough et al., 2007). Furthermore, the reduction of affective labour to labourers would not acknowledge that affect is as much about the capacity to affect as it is about the capacity to be affected. Insofar as the two previous conceptualisations of affect rely on unidirectional notions of capitalist production, they fail to account for multidirectional processes that elude the idea of causal relationships. This article opens up ways to think about how human and non-human bodies and objects become entangled in the processes of affective labour and atmospheres. 
The article proceeds as follows. We lay the groundwork by discussing the capitalisation of affect as a significant cultural transformation in contemporary capitalism. As part of this shift, consumers coparticipate in many forms of affective labour; this complicates previous discussions that have tended to reduce affective labour to new forms of paid employment. Next, we discuss our ethnographic orientations to the exploration of two commercial events. Moving into the analysis, we then demonstrate that many aspects of affective labour cannot be grasped by the individualistic frameworks drawn up by empirical studies of affective labour that focus on human labourers and their intentional work. The co-production of atmospheres might be difficult to recognise as labour at all, yet it is a form of labour that is crucial to the capitalisation of affect. We also identify the limits of intentional planning in the creation of atmospheres: atmospheres work in/through us, but they are not experienced in shared ways, and they can be 'resisted'. This also highlights how atmospheres also come to matter as means of inclusion and exclusion. We conclude that affective labour is best understood as collective labour that takes both intentional and nonintentional forms, across on-site and online processes.

Affective and emotional labour

In late modern Western societies, the capitalisation of affect reflects a shift, from the production and sale of material artefacts and objects, to the production and sale of experiences and affects. Moreover, the experience of affect, rather than the product itself, has also become the most important selling point in a variety of practices that address materially sated consumers (Davies, 2015; Skeggs, 2005: 971). Whereas earlier production processes and industrial work were connected to the production of material objects, contemporary commodities increasingly take the form of information, service, care, communication or affect, and their production thus requires forms of immaterial labour (Hardt, 1999; Hardt and Negri, 2000, 2005; Lazzarato, 2004). While it can be claimed that in the 19th century capitalism had already become oriented towards consumer affects and desires (Davies, 2015), a more recent shift is reflected in the way in which affects and emotions have now become directly productive: they are harnessed and shaped by capital as part of the increasing commodification of 'life itself' (Jokinen, 2010; Skeggs, 2010: 30). The production of atmospheres is an essential form of the capitalisation of affect, which requires labour in order to emerge.

The concept of affective labour describes aspects that many feminist scholars have theorised as emotional and embodied labour, such as waged and unwaged forms of care, reproduction and service (e.g. Hochschild, 1983; Murray, 2015). The conceptualisation of such labour as affective or immaterial, however, has met with feminist critique. Federici (2008), for instance, claims that the concept of affective labour remystifies women's emotional and bodily work in a way that loses what feminist analysis has had to say about the sexual division of labour and the role of reproductive labour in capitalism. Bolton (2009) suggests that the concept of affective labour masks the materiality of women's work and makes women disappear from view, while Fortunati (2007: 147) argues that Hardt and Negri's discussion of affective labour leaves the conceptualisation of affects unclear and risks reducing women to the body. McRobbie (2010) remarks that theorisations of affective labour fail to knit gender as well as ethnicity together with class and class struggle. These concerns are part of a wider feminist critique concerning the highly influential theoretical frameworks of precarity and post-Fordist production, as well as the various empirical studies that operate within those frameworks.

While we are aware of and sympathise with these critiques, we see the value of the notion of affective labour in the examination of atmospheres. In particular, we find its emphasis on the cooperative and nonindividual aspects of labour useful (Hardt and Negri, 2000: 294; Lazzarato, 2004). Likewise, it enables the mobilising of recent theorisations of affect in empirical analyses of labour that falls outside waged work as well as traditional domestic and care work. As Oksala (2016) notes, the notion of affective labour also allows us to understand that capital today does not necessarily subsume and organise labour, but rather 
captures and expropriates common wealth such as affects. Furthermore, since atmospheres cannot be reduced to single affects, we wish to advance an understanding of the capitalisation of affect that extends beyond the idea that affects are detached products that can be identified, manufactured and sold as separate entities.

Affective labour as a concept also highlights aspects that are crucial for our argument but are downplayed in concepts such as emotional labour. Perhaps the best-known feminist theorisation of gendered forms of waged labour is Hochschild's (1983) pioneering theory of emotional labour. According to Hochschild, emotional labour requires one to induce or suppress feeling in order to sustain the outward countenance that produces the proper state of mind in others. This may estrange or alienate the labourer from an aspect of the self - either the body or the margins of the soul - that is used to do the work (Hochschild, 1983: 7). As Weeks (2007: 245) points out, despite its undeniable contributions, this critique of reproductive and emotional labour depends on a critical standpoint located in an outside: in a site separate from capitalism proper, or in a model of the self prior to its estrangement. Using an approach that conceptualises affect beyond subjective feeling, we hope instead to recognise forms of capitalist co-production that are best characterised as trans-subjective or intercorporeal. Differentiating affect from emotion becomes theoretically important here, as it is the concept of affect that invites attention to non-subjective aspects of labour (Wissinger, 2007: 259).

\section{Exploring atmospheres through ethnographic methods}

We ground our analysis in two empirical case studies. In much existing research, atmospheres are discussed either purely theoretically, or as given elements of our experience that are only partially theorised (Sumartojo and Pink, 2018: 15). The social-scientific exploration of atmospheres is challenging, since atmospheres are fleeting and barely perceptible (Bille et al., 2015). Consequently, social-scientific studies of atmospheres have been scarce, and the study of atmosphere has mostly been philosophically inclined (Bille et al., 2015: 33; Schroer and Schmitt, 2018). Human geography is the most notable exception, and the study of atmospheres is an emerging field within the discipline (e.g. Bille et al., 2015; Pink et al., 2018; Pink and Mackley, 2014). Research in human geography has focused on how atmospheres are made during public and collective events (Sumartojo and Pink, 2018: 28). This is also our focus in our case studies in this article.

We consider ethnographic methods especially suitable for the study of atmospheres. We work with a nonhuman-centred concept of atmosphere, one that does not start with an ' $l$ ' but reaches beyond singular, bounded subjects (Kolehmainen, 2019). Atmospheres reach beyond autonomous human subjects, even when they become felt individually, and belong to collective situations (Anderson, 2009; Kolehmainen, 2019; Schroer and Schmitt, 2018; Seyfert, 2012). While atmospheres reach beyond the human subject, their examination benefits from an ethnographic orientation that foregrounds the researcher. Researchers and participants alike are makers and perceivers, (mis)interpreters and co-creators of atmospheres (Schroer and Schmitt, 2018: 6; Sumartojo and Pink, 2018: 40). This refers not only to intentional and conscious activities, but also beyond. By analysing our fieldwork experiences in detail, we offer insights into how the experience of atmospheres can be recognised and mobilised in ethnographic research.

In practice, part of our ethnographic orientation to atmospheres involved attuning to how atmospheres were registered in/through our bodies and focusing on our own affects, senses and sensations.

Nevertheless, our aim was not to centre our own experiences. Rather, we felt (with) our fields through our bodies, in order to get to the affective economy in play in these events (Evers, 2006: 239; Kolehmainen, 2019). This form of enquiry extends beyond mechanical attempts to identify the different elements that make an atmosphere. It is not only the practices of designing and arranging material spaces and objects that are indispensable for creating atmospheres; intentional and unintentional affective encounters and 
entanglements are no less indispensable. Of course, various practices - from the creation and arrangement of light, sounds and symbols, to interior and set design - are crucial to the engineering of atmospheres (Anderson, 2009). However, atmospheres should not be reduced to these practices, as that would posit atmospheres as pre-existing the people who experience them (Schroer and Schmitt, 2018). We have therefore chosen to foreground our own affective states as a way to work with atmospheres.

Our argument is based on two case studies that shed light on the various overlapping processes involved in creating atmospheres through affective labour. Both of the case studies focus on Finland. The first is taken from a study of therapeutic cultures with a focus on relationship and sex counselling practices (Kolehmainen, 2018), and discusses a relationship enhancement seminar. The second comes from a study of professionalised 'mon blogging' (Mäkinen, 2018) and discusses an influencer marketing event. We decided to write an article together after discussing our fieldwork experiences: there seemed to be many overlaps between the two events. For example, similar techniques were mobilised to induce certain atmospheres, even though the events themselves differed. The relationship enhancement seminar was a rather small-scale event with a focus on intimate issues, and the organisers seemed to invest heavily in an entertaining and interactive atmosphere. The influencer marketing event was a relatively large event with a commercial emphasis, and social media played a big role. Interestingly, both events were organised in hotels in the Helsinki metropolitan area, which seemed to blur the boundary between work and leisure - a process in which atmosphere perhaps played a role.

The first author attended the relationship enhancement seminar in 2016. This all-day event featured four different speakers. None of the experts or coaches were certified couple counsellors or therapists, yet each expert speaker took a unique theme for their talk, such as relationship rules and handling finances as a couple. In addition to listening to the talks, the audience was engaged in several ways: in couple discussions, through physical movement, with the suggestion that they prolong the event into a date night in a hotel, and through the distribution of exclusive chocolates. The event was open to both couples and singles. There was an attendance fee of approximately $€ 60$. The researcher attended the event without her partner, and therefore conducted the couple exercises with a (single) woman who was previously unknown to her. Most attendees seemed to be (presumptively) heterosexual couples who were participating in the event together.

It became evident that the speakers and organisers were invested in creating an experience, and not just in providing relationship advice. Even at the beginning of the seminar, it was explicitly stated that the event was supposed be fun, and that all the talks had a humorous undertone. The speakers employed self-irony, embedded their own experiences in the advice given, sought to interact with participants during and outside their talks and so on. The event featured a playlist, which seemed carefully chosen. Before their performances, each of the four experts was introduced in tones of admiration, which was an attempt to make the participants see them in a positive light - and perhaps also to prepare a certain atmosphere for the speaker. All in all, it can be concluded that the atmosphere was not simply there, waiting for participants to experience it (Wetherell, 2012): the organisers used different means to engage the audience and produce an entertaining atmosphere.

The second author attended the full-day influencer marketing event in 2017. 'Influencer' is a relatively new concept that refers to social-media professionals or semi-professionals who use their channel for commercial purposes. In influencer marketing, bloggers, YouTubers and Instagrammers cooperate with marketing agencies and businesses to produce commercial content and reach targeted audiences. The event in question was intended both for content producers (dubbed 'content gurus' on their nametags) and for the business side of the equation ('business hippies' on their nametags). An annual occurrence, and unique in kind, it was a popular event, with approximately 800 participants. For content producers the event was free, whereas for others the cost was over $€ 600$. The author participated in the event as a paying 
'business hippie' (after she contacted the organisers and explained the purpose of her attendance as a researcher, the price was slightly reduced) and took part in keynotes, lunches, networking opportunities and the evening party.

For influencer marketing, social-media presence is key, and so it proved for this event. Recording the event was strongly encouraged on the event's website, and many of the content providers who attended posted their photos, videos and reflections online, either during or after the event. Such social-media documents and narratives mediated the event for those who did not attend, but they were also part of the experience of the event itself. Hence, in addition to attending the event, taking notes, engaging with the activities and talking to participants, the ethnographic orientation in this case consisted of following virtual traces. As a result of this tracing, 21 online documents were collected for closer inspection. These included YouTube videos and promotional texts from the event organisers' website. Posts by various bloggers who attended the event were especially important for the purposes of this article, as they provided insight into the experiences and feelings of attendees. For the purposes of anonymity, and because of copyright issues, however, we do not provide any direct quotes from this material.

\section{Co-production of atmospheres}

During our fieldwork, we noticed that organisers and participants alike invested in creating atmospheres. In other words, affective labour was co-produced in order to maintain an atmosphere. Furthermore, it struck us that obvious investments in atmospheres were an inextricable part of the events we attended. The atmospheres, then, did not just emerge by themselves; rather, their production was explicit, to the extent that the anticipation, expectation and intention of investing in atmospheres actually became part of the atmospheres themselves. There was no clear distinction between the production and consumption of the product. Indeed, our fieldwork highlights that this kind of affective labour, in which the distinction between producer and consumer becomes blurred, is pivotal to various cultural practices, on-site and online.

In the relationship seminar, each expert encouraged interaction - between expert and audience, between participating partners, and even between audience members who were previously unknown to each other. For example, one of the speakers used several ways to activate the audience during his talk. He made participants jump up and down, shake hands with other attendees and embrace their partners:

The speaker urges us to shake hands with somebody previously unknown to us and to thank them for being here. We do as he tells us. After that, he says: we did something in a different manner, and the whole atmosphere changed.

At one point he instructs us to give a hug to our partner. I give a hug to the woman with whom I completed the earlier task. (Relationship seminar, 2016)

He also entertained the audience with his humorous remarks:

He asks something [we are supposed to raise our hands if we want to give an affirmative answer], and then: 'how many of you would not have raised your hands, no matter what I asked?' (Relationship seminar, 2016) 
The speaker was the third out of four speakers during a day-long seminar, and at least for the author, who was participating in the seminar and who had a number of ambivalent feelings and thoughts throughout the day, towards the end it became somewhat challenging to keep on concentrating and feeling enthusiastic, even though she found the event interesting. This reminds us that perhaps the integration of interactive playful elements was also a means to rhythmically coordinate the practices of uplifting or intensifying the atmosphere: an attempt to keep up the spirit.

In any case, activating the audience played a significant role in the creation and crafting of the atmosphere. Through this intentional activation, the audience was invited to do work to produce the atmosphere sought by the organisers. This was confirmed by the speaker himself: 'we did something in a different manner, and the whole atmosphere changed'. The event thus spoke of the need to understand atmospheres as reaching beyond physical space or material place: evidently, much human labour - performed here by both the expert and the participants - was invested in creating a certain atmosphere.

The influencer marketing event was above all a networking event, and the day was full of lectures and workshops on central issues in influencer marketing. The expectation was that attending the event would make sense in terms of one's career or business. Yet the participants were also working for the purposes of the event itself, and for the expectations that had been set for the event to be successful. In particular, participants were responsible for making the event great by taking part in the creation of a specific atmosphere. This was explicitly stated by the organisers in a blog post on the event website:

The blog post states that at this event, the audience is an 'active agent', a 'living organism' that 'affects itself' and creates the atmosphere. The blog post also spells out that the responsibility of any event organiser is to communicate their expectations to the audience. (Influencer marketing event, 2017)

Creating the right atmosphere was thus a crucial part of the influencer marketing event. The invitation to the audience to participate in the creation of the atmosphere was communicated not only verbally but also, for instance, through the display of embodied affects. The event hosts were visibly moved to tears, for example, in a way that sought a specific affective response from the audience as well. For the author attending the event, this open display of embodied reactions was striking. While it almost felt like an 'overthe-top' performance of affects, it served to emphasise the exceptionality of the experience that the event offered, as well as the anticipation that the audience might be similarly moved.

As this event took place not only in the hotel but also across networked social media, the invitation to take part in the production of atmospheres extended to the digital sphere. As already noted, participants were directly encouraged to create and share online content:

Everyone is given a small notebook, in which it says 'share your best [event] moment', and it is promised that the hotel will reward the best pics shared on Instagram or Twitter and hashtagged with [event name] and [hotel name]. In the notebook, we are encouraged: 'have fun. Go crazy'.

I have never seen a buffet table photographed so much. (Influencer marketing event, 2017)

The event's atmosphere was thus connected to social media in two ways. First, the atmosphere was created through the online content, as the participants were simultaneously present at the event and 
following and participating in real-time online documentation. Second, the atmosphere was partly built on the expectation and encouragement of the documentation of the event. As is typical of social media, this online documentation was not merely factual or about recounting events as they unfolded. What was documented and produced through the documentation was also, and importantly, the atmosphere, and the feelings and reactions it created in the participants. Instagram photos from the event, for instance, show smiling people hugging or standing with their arms around each others' shoulders, people posing with funny accessories and peaceful views from the hotel's rooftop swimming pool.

This example pinpoints how the documentation of an ongoing event - and specifically, the expectation and encouragement of that documentation - is crucial for the atmosphere. It thus speaks of the importance of the entanglements between social media, atmosphere and affective labour. For example, the food served at the event was colourful and beautifully laid out, as if waiting to be photographed. Furthermore, taking selfies was encouraged by such settings and with the help of carefully chosen material objects, such as pink balloons that bore the words 'you are awesome' and the hotel logo. These balloons were used intensively as props in pictures, and they were clearly intended for precisely that use. Another example was drawings on the bathroom mirrors. These drawings were designed to look nice in mirror selfies, and they featured in many photos from the event. All of this became part of an atmosphere in which an intensive social-media presence - and also, importantly, the labour that goes into creating that presence - was anticipated. For the author participating in the event, this anticipation felt very distinct and forceful. There was no possibility of being mistaken about what was expected of the participants; the expectations were so clearly spelled out that they felt it almost as a duty to be fulfilled in order to successfully participate in the event.

Both events, then, sustained an atmosphere whose creation through encouragement on the part of the organisers was a component of the atmosphere itself. The audiences were intentionally invited to do work for the sake of the atmosphere - and the invitation to do that work was presented both through explicit discourse, as with the relationship enhancement seminar, and through material settings, as with the influencer marketing event. The work the participants did on-site and online was concrete. However, since the outcome of that work was not material objects but the production of an atmosphere, the work is best described as affective labour: labour that produces affective intensities and encounters, and thus contributes to the creation of atmospheres. Affective labour, when understood through a relational and non-individual notion of affect, is about contacts and encounters - in the first example, through the physical intimacy of human bodies, and in the second, through virtual practices that also had material connections and contexts. Hence, the co-creation of atmospheres, and the labour invested in that process, cannot be understood as limited exclusively to individual, autonomous and bounded human subjects.

Intercorporeal and trans-subjective crafting of atmospheres

Although the previous section stressed multiple co-production processes, this is not to say that the participants in these two commercial events necessarily recognised their own active roles, or that they as individuals would intentionally or knowingly invest in the crafting of atmosphere. Rather, through encouragement by means of discourse and material settings, they became part of a collective and embodied co-production or co-creation of atmospheres. Indeed, exploring the emergence of atmospheres with the help of affect theory enables us to pay attention to how bodies affect and become affected in ways that emphasise those bodies' intercorporeality and trans-subjectivity.

In both events, participants were encouraged to engage in interactive settings and go with the flow - even to have fun. Although participation was voluntary, participants' actions were an integral part of the event design: their share in the course of events was essential. For example, human contact and the subsequent exchange of relationship experiences not only produced a relaxed atmosphere, but was also a way to integrate participants' experiences into the knowledge economy of the relationship seminar. The following 
example illustrates that interaction with the other attendees was a crucial activity, perhaps with the idea that mingling makes the atmosphere more relaxed but also so that the attendees could learn from each others' relationships and experiences. The example also highlights how the event evoked different affective resonances in the three attendees, as two of them found the financial theme very relevant, unlike the author participating in the event, who personally was not similarly impressed about this:

The next task is to be conducted in pairs of two women or two men. I discuss with a woman sitting behind me, and with another woman who has been sitting with her partner in the same row as me. The younger of the two says that they have a toddler, and for a long time she held the view that people could not visit their home if it was messy. However, one time she did not clean up [before a friend came to visit], and it was a relief when the friend stated that it was lovely to see that not everyone's home was always in tip-top condition. [. . . ] It also becomes evident that both the women have found the talk about finance interesting and fruitful. (Relationship seminar, 2016)

As the example demonstrates, during the relationship seminar, the attendees were actively made to interact not only with their partners but also more and more with strangers - especially towards the end of the day. If the first contacts with the fellow attendees involved shaking hands and uttering a few words, by the end of the seminar, we were supposed to share more intimate thoughts and experiences. The attendees were grouped in different ways during the day, as well as being made into a single collective. In this way, personal boundaries were blurred, and proximity between human bodies was encouraged, but as this happened step by step the greatest discomfort was prevented. Historically, the mass has been associated - from a middle-class perspective - with feminised metaphors of fluidity and suggestibility, and the blurring of boundaries between self and other has been seen as a threat to middle-class individuality (Swan, 2008: 95). However, in the course of the relationship seminar, the boundaries between self and other appeared more complex. The participants' experiences formed part of economic co-production, as their intimate experiences were converted into 'capital' and integrated into capitalist supply chains (Tsing, 2015). It is also noteworthy that the participants were supposed to form pairs on the basis of their (assumed) gender. Gender thus links to the processes of capitalist accumulation, as experiences categorised as 'male' or 'female' are converted into expert knowledge.

At the influencer marketing event, the above-mentioned idea of the audience as a living organism was materialised, for instance, through a clapping performance, during which audience members clapped their own body parts and each other's knees. This performance too transcended the boundaries of individual bodies and blurred personal boundaries, turning the audience into a collective in a very embodied way:

The audience was invited to first clap their hands in sync, and then clap their knees, and finally also to clap each other's knees, all in sync and led by a performing artist. This was a performance that started the event, and it set the scene for the audience to feel and move in sync. (Influencer marketing event, 2017)

While the author attending this event felt somewhat confused and clumsy in trying to blend in and keep up with the clapping as the patterns became more and more elaborate, the surrounding members of the audience were smiling and seemed to be enjoying their opportunity to participate in the performance. 
Atmospheres can be made more intense by creating patterns of affective imitation (Anderson, 2009), and here clapping in sync is a way to intensify an atmosphere. At the same time, this illustrates that atmospheres are not psychological, individual states of mind; they do not come from 'inside' - nor do they simply reside 'outside' the subject (Bille et al., 2015; Seyfert, 2012). At the event, rhythmic touch and the emerging sound pattern turned individual bodies into a collective body, almost literally. This speaks of the relevance of intercorporeality and trans-subjectivity when one is exploring affective labour in the crafting of atmospheres. Furthermore, it underlines the ways in which labour - in a context of 'economic experimentation with the creation of monetary value out of knowledge/culture/affect' (Terranova, 2000: 38) - is not necessarily a possession that individual workers intentionally exchange for a wage, but works across intercorporeal and trans-subjective affects.

Hence, atmospheres are produced through collectives in which the boundaries between individual bodies are not necessarily maintained, and in which affective experiences extend beyond individual emotional states. Doing affective labour might not look like doing labour at all, and may be more like being part of a collective event. At the events we attended, it seemed natural that organisers and attendees would prefer a cosy and relaxed (or otherwise good) atmosphere over its alternatives. A nice atmosphere would 'benefit' organisers and attendees alike. Understanding these kinds of collective experience as forms of affective labour, then, does not imply that they cannot be genuinely empowering, or at least pleasing, for the participants. But the 'benefits' of the atmosphere do not mean that no work is involved in the creation of that atmosphere. These notions also pinpoint that - contrary to theories that see emotional labour as a burden that results in estrangement - emotional or affective labour is not necessarily experienced as conflicting with the labourer's own interests.

Belonging and non-belonging: limits of inclusion

In the previous sections, we highlighted how organisers and attendees at commercial events participate in forms of affective labour in the production of atmospheres, and how that labour is embodied and collective, stressing the importance of the intercorporeal and trans-subjective. The creation of atmospheres through such co-productive affective labour, however, can be tricky. Especially in a commercial setting, the expectations set by organisers, for example, can easily feel manipulative or oppressive. The crossing of bodily boundaries does not necessarily create connections between bodies, but can instead feel intrusive, thereby creating a heightened sense of those boundaries. The display and creation of intense affective reactions can also appear uncomfortable, even ridiculous, to anyone who for some reason does not catch those affective states. For instance, in the case of the clapping performance described above, one participant later mentioned on her blog that she was simply unable to participate in that kind of play. This reminds us that atmospheres cannot make people feel particular things, precisely because it is the way people feel about things that makes atmospheres perceptible (Sumartojo and Pink, 2018).

Co-produced atmospheres can also heighten the dynamics of inclusion and exclusion. If the atmosphere is central to the experience of an event, and if it is co-created and co-produced through embodied contacts and reactions and through material settings and objects, not feeling the right thing means not really being part of the event. If feeling the right thing is part of the expectation for the audience or participants, then not feeling that thing can be experienced as failure. Some participants in the influencer marketing event described such experiences on their blogs afterwards. A moment during the influencer event's evening party, recalled by the second author, illustrates both the expectations of co-productive atmosphere creation and the dynamics of inclusion and exclusion:

I was feeling a bit left out, since I didn't know anyone and no one came to talk to me. I was wandering around and had a few drinks [both alcoholic and non-alcoholic], and I followed the award ceremony for 
best outfits, and then there were some games. After that, I stood alone, and was browsing the Internet on my phone or something like that. Suddenly one of the organisers came up to me and said in an overly chirpy and pushy manner, 'is there a party here somewhere? Is there a party? Are we having fun!?' Then they went away. I was very confused, and experienced this really as a reproach: I was not having fun, so I did not fit the party. After this, I soon went home. (Influencer marketing event, 2017)

Such experiences show how, in commercial settings, co-productive affective labour is not merely organic or freeform, but may be carefully designed and even disciplined. The exhortation to have fun illustrates how 'life is made to work for production and production is made to work for life' (Hardt and Negri, 2000: 32) at such events, in an upbeat and enjoyable way. Yet it also reminds us that affective labour is not only about creating connections, it is also about the simultaneous creation of disconnections and exclusions.

Likewise, the relationship enhancement seminar entailed situations in which investments in the cheerful atmosphere were greeted with discomfort. The organisers, including the hosts, had invested heavily in creating an engaging, uplifting atmosphere. For example, when the speakers were introduced, they were praised, songs were played whose themes matched the speakers' topics and the speakers entered the stage only after some careful 'warming up':

A woman introduces the next speaker. I find her presentation forced, and she uses the word 'fantastic' and other praising terms. When the speaker approaches the stage, they play a song by a famous Finnish rap artist until the speaker enters the 'stage' - it is not even a real stage; he enters the front of the room only. I recognise the song and actually have listened to it sometimes, yet I am somewhat confused, as in my circles the song would be interpreted as a sign of 'bad taste'. (Relationship seminar, 2016)

The atmosphere here did not quite 'catch' the author, who found that the distance between the organiser(s) and speaker on one side and the audience on the other was highlighted - more made than unmade. The speaker was introduced as if he were a big star entering a huge stage, which felt awkward and unnecessarily hierarchical. The author also saw the host's demeanour as non-genuine. This kind of fieldwork experience also highlights that genuineness and authenticity are desired features in the assessment of atmospheres (Bille et al., 2015). Although there are no 'authentic' atmospheres (Carlson and Stewart, 2014: 127), the impression of inauthenticity easily contributes to the intensification of a feeling of non-belonging. All in all, the organisers' efforts contributed to an imperative to experience the situation in a certain way: atmospheres are also about norms - about what should be felt (Bille et al., 2015) - and here the aim of creating some hype was enacted too forcefully. It was perhaps too obvious that the organisers had made preparations, and their labour was 'revealed' through the attempts to sustain a nice atmosphere.

Both examples demonstrate that the attempt to create an uplifting atmosphere, and to make the audience feel good or entertained, paradoxically made the investment in atmospheres - and the related affective labour - too tangible. Even though the creation of atmospheres requires affective labour, this labour should not become too observable, but should be experienced as 'natural' or free-flowing. The moment this labour, or the expectation of such labour, becomes too visible or forceful is thus a moment when it fails. However, such failures perhaps stress that people's experiences cannot be endlessly 'manipulated', no matter how much organisers invest in creating certain kinds of atmosphere. As became evident in our fieldwork experiences, affect resists prediction and manipulation, and as such it 'emerges in ways that cannot be controlled by the technologies that bring it into effect' (Skeggs, 2010: 48). What we experienced 
during our on-site participant observation as the failure of atmospheres to catch us also directs attention to the limits of the capitalisation of affects.

\section{Conclusion}

In this article, we have explored the ways in which atmospheres are brought into being - created, designed and experienced - in commercial settings. Crucial to our discussion is the recognition that atmospheres are produced through affective labour in processes that extend beyond the individual. We therefore argue that affective labour is not best understood as individual labour alone: it takes multiple forms, and many of these are collective and go beyond the individual's emotional or other investments. Our analysis has also shown that the creation of atmosphere demands co-productive labour that is affective, embodied and interactive, and that involves encounters not only among humans but also with materialities such as food, balloons, mirrors and notebooks, and networked affects. We have also shown that audiences or participants are actively involved in the production of atmospheres, both intentionally and unintentionally. The co-production of atmospheres not only articulates this new kind of labour, but also highlights how the co-production of atmospheres is itself a component of atmospheres.

What follows from our discussion is the need for research to acknowledge that affective labour is not always something that workers do for their clients. The interests of workers and clients (or audiences) do not necessarily differ on every occasion - or at least, they are not experienced as differing. This also needs to be acknowledged in studies of affective labour, especially when affect is seen to refer to a wider phenomenon than human emotion alone. Furthermore, what can be seen as 'work' or 'labour' gets blurred: it is not simply that work and leisure overlap, but rather that paying customers actively participate in producing communal feelings that can be capitalised. Affective labour blurs the boundaries not only between work and leisure, but also between workers and clients.

The creation of atmospheres also orchestrates social inclusion and exclusion (Bille et al., 2015: 36), although this does not require any conscious intention. As our analysis has shown, the atmospheres did not always catch us. An atmosphere is already angled; it is always felt from a specific point (Ahmed, 2010: 37). The ways in which gender, sexuality, class, race or ability relate to the dynamics of inclusion and exclusion are beyond the scope of this article. Yet our observations invite further enquiry into how intersecting power relations might be enforced in the context of affective labour and the production of atmospheres. Overall, thinking atmospherically about collectivity and power opens up new routes not only into insights about inclusion and exclusion, but also to the points where these might be changed or developed, and the best ways to do this (Sumartojo and Pink, 2018).

We have sought to understand affective labour in settings that are clearly commercial, but where economic processes are not linear in a straightforward sense of 'products' being made by producers and bought by consumers. In the case of events like those discussed above, the concept of affective labour can open up the ways in which capital captures affects and puts them to work without directly controlling or organising labour power. At both events, the production of atmospheres was orchestrated partly to serve commercial interests. For instance, sharing pictures from the influencer marketing event was also part of the marketing campaign of the hotel that hosted the event. Even if the participants were not paid to work to produce atmospheres - on the contrary, they were themselves paying - they were nevertheless contributing to commercial goals. This is certainly telling of the ways in which capital now seeks to benefit from affects associated with uncommercial social bonds, such as friendships, and of the ways in which digital media allow for the tracking and calculation of such affective bonds (Davies, 2015), in this case, for instance, through the pictures that were shared from the event. The affective labour that the audiences were invited to perform, then, can be seen as a form of labour that took place within a decentred production process; it had not developed in response to the needs of capital in any direct cause-and-effect fashion, but it 
nevertheless had value and could be harnessed for capital. This kind of 'free cultural/affective labour' (Terranova, 2000: 38 ) is often not immediately recognisable as labour, but rather is experienced as voluntary and pleasurable - and yet it is central to capitalist production.

Funding

The authors disclosed receipt of the following financial support for the research, authorship, and/or publication of this article: Marjo Kolehmainen's work was funded by the Academy of Finland project Just the two of us? Affective Inequalities in Intimate Relationships (grant number 287983). Katariina Mäkinen's research is funded by the Academy of Finland (project number 326562).

ORCID iD

Marjo Kolehmainen https://orcid.org/0000-0003-2949-2638

\section{References}

Ahmed S (2010) Happy objects. In: Gregg M, Seigworth GJ (eds) The Affect Theory Reader. Durham, NC: Duke University Press. Crossref.

Anderson B (2009) Affective atmospheres. Emotion, Space and Society 2: 77-81. Crossref.

Bille M, Bjerregaard P, Flohr Sørensen T (2015) Staging atmospheres: Materiality, culture, and the texture of the inbetween. Emotion, Space and Society 15: 31-38. Crossref.

Blackman L (2010) Embodying affect: Voice-hearing, telepathy, suggestion and modelling the nonconscious. Body and Society 16(1): 163-192. Crossref.

Bolton SC (2009) The lady vanishes: Women's work and affective labour. International Journal of Work Organisation and Emotion 3(1): 72-80. Crossref.

Carlson JD, Stewart KC (2014) The legibilities of mood work. New Formations 82: 114-133. Crossref.

Clough PT, Goldberg G, Schiff R, et al. (2007) Notes towards a theory of affect itself. Ephemera 7(1): 60-77.

Davies W (2015) The Happiness Industry. How the Government and Big Business Sold Us Well-being. London: Verso.

Edensor T (2015) Producing atmospheres at the match: Fan cultures, commercialisation and mood management in English footfall. Emotion, Space and Society 15: 82-89. Crossref.

Evers C (2006) How to surf. Journal of Sport and Social Issues 30(3): 229-243. Crossref.

Federici S (2008) Precarious labour: A feminist viewpoint. In the Middle of a Whirlwind. Available at: http://inthemiddleofthewhirlwind.wordpress.com/precarious-labor-a-feminist-viewpoint/ (accessed 7 January 2019).

Fortunati L (2007) Immaterial labour and its machinization. Ephemera 7(1): 139-157. 
Hardt M (1999) Affective labour. Boundary 26(2): 89-100.

Hardt M, Negri A (2000) Empire. Cambridge, MA: Harvard University Press.

Hardt M, Negri A (2005) Multitude. London: Hamish Hamilton.

Hochschild A (1983) The Managed Heart: Commercialization of Human Feeling. Berkeley, CA: University of California Press.

Jokinen E (2010) Affektiivinen työ ja sukupuoli. Kulttuurintutkimus 27(2): 44-49.

Kolehmainen M (2018) Mapping affective capacities: Gender and sexuality in relationship and sex counselling practices. In: Juvonen T, Kolehmainen M (eds) Affective Inequalities in Intimate Relationships. London: Routledge, pp.63-78. Crossref.

Kolehmainen M (2019) Affective assemblages: Atmospheres and therapeutic knowledge production in/through the researcher-body. In: Salmenniemi S, Nurmi J, Bergroth H, et al. (eds) Assembling Therapeutics. London: Routledge, pp.43-57. Crossref.

Lazzarato M (2004) From capital-labour to capital-life. Ephemera 43(3): 187-208.

McRobbie A (2010) Reflections on feminism and immaterial labour. New Formations 70: 60-76. Crossref. Mäkinen K (2018) Negotiating the intimate and the professional in mom blogging. In: Luckman S, Taylor S (eds) The New Normal of Working Lives. Cham: Palgrave Macmillan, pp.129-146. Crossref.

Murray JM (2015) Nightlife girls: Envy, theatre, and encounters with men and women. Gender, Work and Organization 22(3): 197-210. Crossref.

Oksala J (2016) Affective labour and feminist politics. Signs 41(2): 281-303. Crossref.

Pink S, Mackley KL (2014) Moving, making and atmosphere: Routines of home as sites for mundane improvisation. Mobilities 11(2): 171-187. Crossref.

Pink S, Hjorth L, Horst H, et al. (2018) Digital work and play: Mobile technologies and new ways of feeling at home. European Journal of Cultural Studies 21(1): 26-38. Crossref.

Schroer SA, Schmitt SB (2018) Introduction: Thinking through atmospheres. In: Schroer SA, Schmitt SB (eds) Exploring Atmospheres Ethnographically. London: Routledge, pp.1-11.

Seyfert R (2012) Beyond personal feelings and collective emotions: Towards a theory of social affect. Theory, Culture and Society 29(6): 27-46. Crossref.

Skeggs B (2005) The making of class and gender through visualizing moral subject formation. Sociology 39(5): 965-982. Crossref.

Skeggs B (2010) The value of relationships: Affective scenes and emotional performances. Feminist Legal Studies 18(1): 29-51. Crossref.

Sumartojo S, Pink S (2018) Atmospheres and the Experiential World: Theory and Methods. Abingdon: Routledge. Crossref.

Swan E (2008) Therapeutic cultures and the contagion of femininity. Gender, Work and Organization 15(1): 88-107. Crossref.

Terranova T (2000) Free labour: Producing culture for the digital economy. Social Text 18(2): 33-58.

Crossref. 
Tsing AL (2015) The Mushroom at the End of the World: On the Possibility of Life in Capitalist Ruins. Princeton, NJ: Princeton University Press. Crossref.

Weeks K (2007) Life within and against work: Affective labor, feminist critique, and post-Fordist politics. Ephemera 7(1): 233-249.

Wetherell M (2012) Affect and Emotion: A New Social Science Understanding. London: Sage. Crossref.

Wissinger E (2007) Modelling a way of life: Immaterial and affective labour in the fashion modelling industry. Ephemera 7(1): 250-269.

\section{Biographical notes}

Marjo Kolehmainen is a postdoctoral research fellow in gender studies at the Tampere University. Her current research explores the practices of relationship and sex counselling. She has authored articles published in, for example, Feminist Media Studies, Sexualities, The Sociological Review, and Body \& Society, and is also a co-editor of Affective Inequalities in Intimate Relationships (Routledge, 2018).

Katariina Mäkinen is currently a postdoctoral researcher in gender studies at the Tampere University. Her research investigates the intersections of gendered work, digitalisation and intensive mothering. 